University of Nebraska - Lincoln DigitalCommons@University of Nebraska - Lincoln

2014

\title{
The effect of general statistical fiber misalignment on predicted damage initiation in composites
}

Brett Bednarcyk

NASA Glenn Research Center, Brett.A.Bednarcyk@nasa.gov

Jacob Abdoudi

Tel Aviv University

Steven Arnold

NASA Glenn Research Center

Follow this and additional works at: http://digitalcommons.unl.edu/nasapub

Bednarcyk, Brett; Abdoudi, Jacob; and Arnold, Steven, "The effect of general statistical fiber misalignment on predicted damage initiation in composites" (2014). NASA Publications. 132.

http://digitalcommons.unl.edu/nasapub/132

This Article is brought to you for free and open access by the National Aeronautics and Space Administration at DigitalCommons@University of Nebraska - Lincoln. It has been accepted for inclusion in NASA Publications by an authorized administrator of DigitalCommons@University of Nebraska - Lincoln. 


\title{
The effect of general statistical fiber misalignment on predicted damage initiation in composites
}

\author{
Brett A. Bednarcyk ${ }^{a, *}$, Jacob Aboudi $^{\mathrm{b}}$, Steven M. Arnold ${ }^{\mathrm{a}}$ \\ a NASA Glenn Research Center, Cleveland, OH, USA \\ ${ }^{\mathrm{b}}$ Tel Aviv University, Tel Aviv, Israel
}

\section{A R T I C L E I N F O}

\section{Article history:}

Received 7 November 2013

Received in revised form 17 April 2014

Accepted 22 April 2014

Available online 2 May 2014

\section{Keywords:}

C. Micro-mechanics

B. Strength

B. Directional orientation

C. Analytical modelling

High-Fidelity Generalized Method of Cells

\begin{abstract}
A B S T R A C T
A micromechanical method is employed for the prediction of unidirectional composites in which the fiber orientation can possess various statistical misalignment distributions. The method relies on the probability-weighted averaging of the appropriate concentration tensors, which are established by the micromechanical procedure. This approach provides access to the local field quantities throughout the constituents, from which initiation of damage in the composite can be predicted. In contrast, a typical macromechanical procedure can determine the effective composite elastic properties in the presence of statistical fiber misalignment, but cannot provide the local fields. Fully random fiber distribution is presented as a special case using the proposed micromechanical method. Results are given that illustrate the effects of various amounts of fiber misalignment in terms of the standard deviations of in-plane and out-of-plane misalignment angles, where normal distributions have been employed. Damage initiation envelopes, local fields, effective moduli, and strengths are predicted for polymer and ceramic matrix composites with given normal distributions of misalignment angles, as well as fully random fiber orientation. Published by Elsevier Ltd.
\end{abstract}

\section{Introduction}

It is well-known that a micromechanical analysis can provide the effective properties of composite materials from the knowledge of the constituent properties, their geometric arrangement, and their detailed interactions. However, the impact of fiber misalignment on the composite response, which often can occur in modern composites [7], is typically not accounted for in design and analysis. A notable exception is the effect of longitudinal fiber misalignment on the compressive failure response of composites, which has been studied extensively in the literature (c.f., $[10,34,37,11,33,35,22,8,31,40,6])$. Such misalignment can occur due to manufacturing deficiency and defects, curved surfaces, and fiber waviness, which was investigated by Kugler and Moon [18]. A standard approach to incorporating the effect of fiber misalignment in a unidirectional composite is to consider the anisotropic ply properties and perform a standard transformation to a desired fiber orientation (for instance, an in-plane rotation, c.f., Jones [17] and Herakovich [14]). While such a macromechanical approach will provide the effective properties of the ply, the associated local field distributions within the constituent materials

\footnotetext{
* Corresponding author. Tel.: +1 (216) 433 2012; fax: +1 (216) 4338300 .

E-mail address: Brett.A.Bednarcyk@nasa.gov (B.A. Bednarcyk).
}

are not available (as is always the case with the macromechanical approach). It is precisely these local fields that dictate and drive the damage initiation and progression, yielding, failure, and other nonlinearities, which impact the macroscopic composite response and the performance of structures composed of composite materials. Alternatively, if one utilizes an appropriate micromechanical analysis, the local field variations within the composite constituents can be predicted based on the knowledge of the strain (or stress) concentration tensors, which are naturally given by the micromechanics theory. Consequently, the effect of fiber misalignment on the local fields in the composite can be captured through the use of these concentration tensors.

In the present investigation, the micromechanical analysis known as the High-Fidelity Generalized Method of Cells (HFGMC) [2] is enhanced to capture the effects of possible fiber misalignment in unidirectional composites. In the latter reference, HFGMC has been shown to provide a reliable and robust micromechanical method for predicting the properties and nonlinear response of a wide array of composites. Further, it was shown that the strain (or stress) concentration tensors are fundamental to predicting both the local and global composite response and thus they are used herein to predict the effect of fiber misalignment on the composite behavior. In particular, the concentration tensors are employed in an averaging procedure to determine the effect of a 
known distribution of misalignment angles (i.e., orientation distribution function), or completely randomly fiber orientation, on the local fields in the composite. Consequently, the initial yield and damage surfaces, field distributions in the constituents, as well as the standard effective properties, can be predicted for the composite.

Averaging based on orientation has been employed extensively within the microsphere model [26], which was originally developed for analyzing rubber $[27,13]$. Since then, the microsphere model has been used extensively in the analysis of biological materials. Menzel and Waffenschmidt [25] used the model, with an evolving orientation distribution function, to simulate remodeling in soft tissues. Murtada et al. [30] used the microsphere approach to analyze smooth muscles, wherein a specialized distribution of the muscle contractile fiber orientation, as a function of stretch, was employed. Alastrué et al. [3] first used a $\pi$-periodic von Mises orientation distribution function, and then a Bingham orientation distribution function [4] in applying the microsphere model to blood vessels. Waffenschmidt et al. [36] used the microsphere approach, with evolving orientation density functions, to model bone remodeling. $\mathrm{Li}$ et al. [21] have recently applied the microsphere approach to structural composite materials, wherein a von Mises fiber orientation distribution has been employed. Orientational averaging was also recently employed by Modniks and Andersons [28] to model the nonlinear mechanical response of short-flax-fiber-reinforced composites using an analytical approach. While the approach and equations were general in terms of fiber misalignment distribution, results focused on a uniform distribution.

The remainder of this paper is organized as follows. A brief description of the HFGMC methodology is provided, followed by details on the incorporation of the statistical fiber misalignment distributions. Results are then given exhibiting the impact of fiber misalignment on the properties, damage initiation envelopes, and critical values of the local field variables. Two classes of composite materials are examined: a polymer matrix composite (PMC) system, namely, graphite/epoxy, and a ceramic matrix composite (CMC) system. While the PMC system exhibits extreme mismatch in properties between the constituents, the CMC system has low mismatch between the fiber and matrix, but includes a very compliant fiber interfacial layer.

\section{High-Fidelity Generalized Method of Cells micromechanical model}

The HFGMC micromechanical model is employed herein to predict the effective behavior of unidirectional composites with known fiber misalignment. This theory has been fully described by Aboudi et al. [2]. The continuously reinforced (i.e., doubly periodic) version of HFGMC is briefly outlined in the following. The doubly periodic microstructure considered is shown in Fig. 1(a) in terms of the global coordinates $\left(x_{2}, x_{3}\right)$. The repeating unit cell, Fig. 1(b), defined with respect to local coordinates $\left(y_{2}, y_{3}\right)$, of such a composite is divided into $N_{\beta}$ and $N_{\gamma}$ subcells in the $y_{2}$ and $y_{3}$ directions, respectively. Each subcell is labeled by the indices $(\beta \gamma)$ with $\beta=1, \ldots, N_{\beta}$ and $\gamma=1, \ldots, N_{\gamma}$, and may contain a distinct homogeneous material. The dimensions of subcell $(\beta \gamma)$ in the $y_{2}$ and $y_{3}$ directions are denoted by $h_{\beta}$ and $l_{\gamma}$, respectively. A local coordinate system $\left(\bar{y}_{2}^{(\beta)}, \bar{y}_{3}^{(\gamma)}\right)$ is introduced in each subcell whose origin is located at its center. The local (subcell) constitutive equation of the elastic, anisotropic material is given by,

$\sigma_{i j}^{(\beta \gamma)}=C_{i j k l}^{(\beta \gamma)} \varepsilon_{k l}^{(\beta \gamma)}$

where $\sigma_{i j}^{(\beta \gamma)}, \varepsilon_{k l}^{(\beta \gamma)}$, and $C_{i j k l}^{(\beta \gamma)}$ are the components of the stress, strain, elastic stiffness tensors, respectively.
The basic assumption in HFGMC is that the displacement vector $u_{i}^{(\beta \gamma)}$ in each subcell is expanded into quadratic forms in terms of its local coordinates $\left(\bar{y}_{2}^{(\beta)}, \bar{y}_{3}^{(\gamma)}\right)$, as follows,

$$
\begin{aligned}
u_{i}^{(\beta \gamma)}= & \bar{\varepsilon}_{i j} x_{j}+W_{i(00)}^{(\beta \gamma)}+\bar{y}_{2}^{(\beta)} W_{i(10)}^{(\beta \gamma)}+\bar{y}_{3}^{(\gamma)} W_{i(01)}^{(\beta \gamma)} \\
& +\frac{1}{2}\left(3 \bar{y}_{2}^{(\beta) 2}-\frac{h_{\beta}^{2}}{4}\right) W_{i(20)}^{(\beta \gamma)}+\frac{1}{2}\left(3 \bar{y}_{3}^{(\gamma) 2}-\frac{l_{\gamma}^{2}}{4}\right) W_{i(02)}^{(\beta \gamma)}
\end{aligned}
$$

where $\bar{\varepsilon}_{i j}$ is the applied (external) average strains, and the unknown terms $W_{i(l m)}^{(\beta \gamma)}$ must be determined from the fulfillment of the equilibrium conditions, the periodic boundary conditions, and the interfacial continuity conditions of displacements and tractions between subcells. The periodic boundary conditions ensure that the displacements and tractions at opposite surfaces of the repeating unit cell are identical. A principal ingredient in the HFGMC micromechanical analysis is that all these conditions are imposed in the average (integral) sense.

As a result of the imposition of these conditions, a linear system of algebraic equations is obtained, which can be represented in the following form:

$\mathbf{K U}=\mathbf{f}$

where the matrix $\mathbf{K}$ contains information on the geometry and properties of the materials within the individual subcells $(\beta \gamma)$, and the displacement vector $\mathbf{U}$ contains the unknown displacement coefficients $W_{i(l m)}^{(\beta \gamma)}$, which appear on the right-hand side of Eq. (2). The vector $\mathbf{f}$ contains information on the applied average strains $\bar{\varepsilon}_{i j}$. The solution of Eq. (3) enables the establishment of the following localization relation which expresses the average strains $\bar{\varepsilon}_{i j}^{(\beta \gamma)}$ in the subcell $(\beta \gamma)$ to the externally applied average strains $\bar{\varepsilon}_{i j}$ in the form,

$\bar{\varepsilon}_{i j}^{(\beta \gamma)}=A_{i j k l}^{(\beta \gamma)} \bar{\varepsilon}_{k l}$

where $A_{i j k l}^{(\beta \gamma)}$ are the strain concentration tensor components, of the subcell $(\beta \gamma)$. The final form of the effective constitutive law of the multi-phase thermo-inelastic composite, which relates the average stresses $\bar{\sigma}_{i j}$ and strains $\bar{\varepsilon}_{k l}$, is established as follows:

$\bar{\sigma}_{i j}=C_{i j k l}^{*} \bar{\varepsilon}_{k l}$

In this equation $C_{i j k l}^{*}$ are components of the effective stiffness tensor, which are given by,

$C_{i j k l}^{*}=\frac{1}{H L} \sum_{\beta=1}^{N_{\beta}} \sum_{\gamma=1}^{N_{\gamma}} h_{\beta} l_{\gamma} C_{i j p q}^{(\beta \gamma)} A_{p q k l}^{(\beta \gamma)}$

Next, the components of stress concentration tensor, $B_{i j k l}^{(\beta \gamma)}$, which relate the average stresses in the subcell, $\bar{\sigma}_{i j}^{(\beta \gamma)}$, to the average (global) stresses, $\bar{\sigma}_{i j}$, are determined. By combining Eqs. (1) and (4), the subcell stresses are given by,

$\bar{\sigma}_{i j}^{(\beta \gamma)}=C_{i j p q}^{(\beta \gamma)} A_{p q k l}^{(\beta \gamma)} \bar{\varepsilon}_{k l}$

Then, using Eq. (5), one obtains,

$\bar{\sigma}_{i j}^{(\beta \gamma)}=B_{i j k l}^{(\beta \gamma)} \bar{\sigma}_{k l}$

where

$B_{i j k l}^{(\beta \gamma)}=C_{i j p q}^{(\beta \gamma)} A_{p q r s}^{(\beta \gamma)} S_{r s k l}^{*}$

and $S_{r s k l}^{*}$ are components of the effective compliance tensor.

Consequently, with the present information provided by the HFGMC, the local elastic fields throughout the composite constituents are known, as are the composite effective (homogenized) properties. 


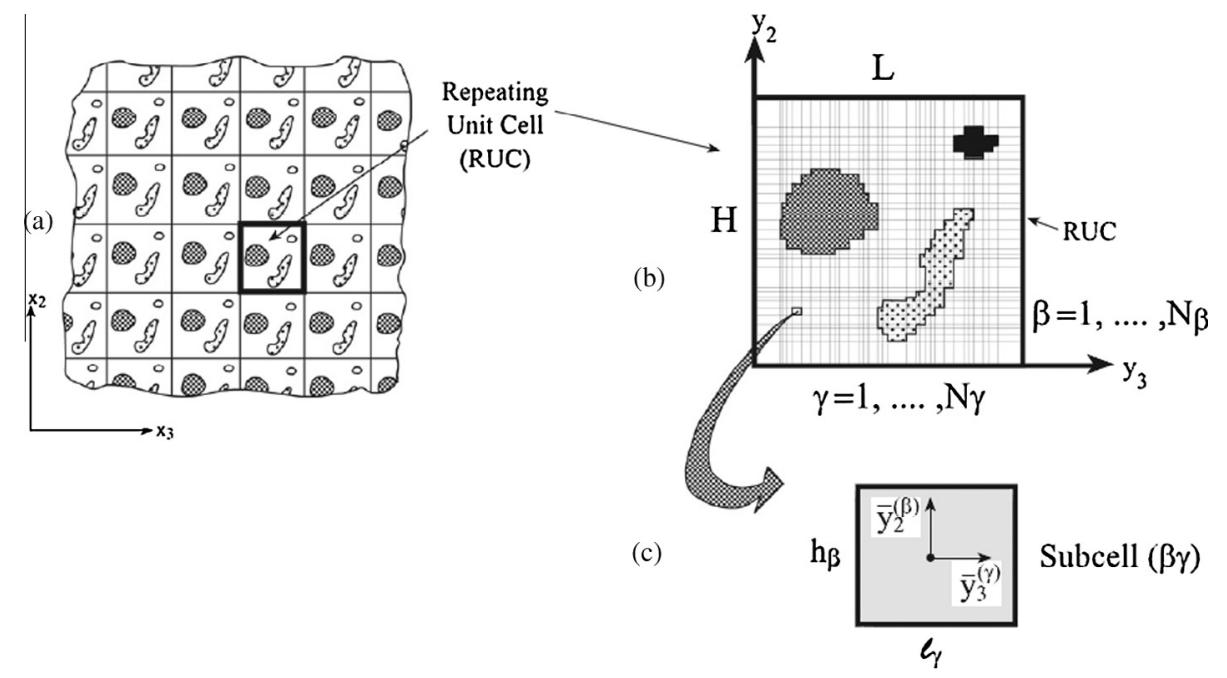

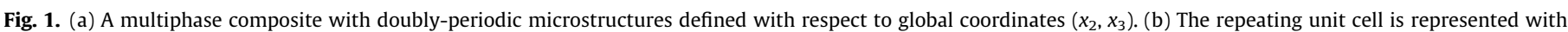

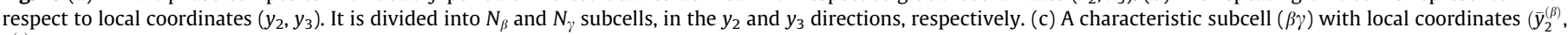
$\left.\bar{y}_{3}^{(\gamma)}\right)$ whose origin is located at its center.

\section{Incorporation of fiber misalignment distribution}

\subsection{Transformation to misalignment angles}

Suppose that the fibers of the unidirectional composite are oriented in the $x_{1}$-direction. In order to express a rotation of the fiber direction by angle $\psi$ in the $x_{1}-x_{2}$ plane about the $x_{3}$-axis (see Fig. 2), the following transformation matrix must be employed,

$\boldsymbol{\Psi}=\left[\begin{array}{ccc}\cos \psi & -\sin \psi & 0 \\ \sin \psi & \cos \psi & 0 \\ 0 & 0 & 1\end{array}\right]$

Subsequently, a rotation about the new $x_{2}$-axis, namely $x_{2}^{\prime}$, by an angle $\phi$ is performed (see Fig. 2) which can be expressed by the following transformation matrix,

$\boldsymbol{\Phi}=\left[\begin{array}{ccc}\cos \phi & 0 & \sin \phi \\ 0 & 1 & 0 \\ -\sin \phi & 0 & \cos \phi\end{array}\right]$

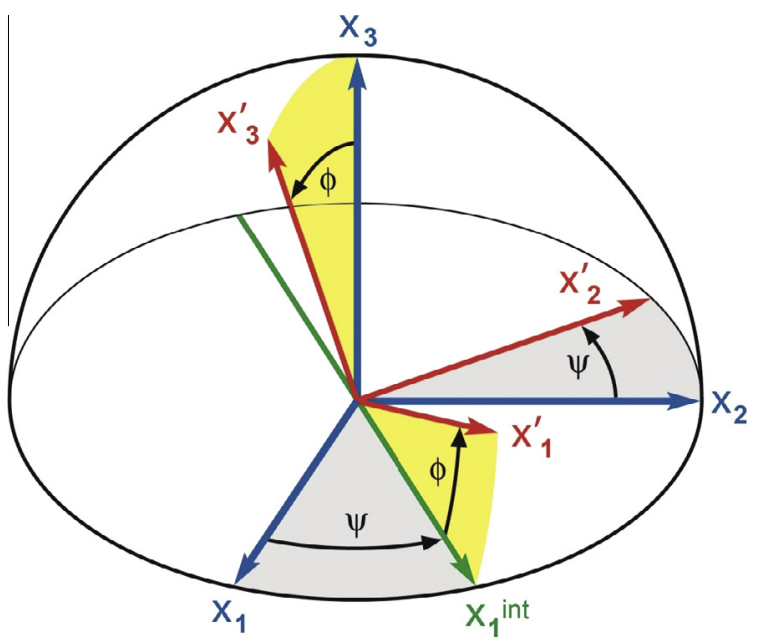

Fig. 2. Transformation from the original (un-prime) to rotated (prime) coordinate system through the rotation angles $\psi$ and $\phi . x_{1}^{\text {int }}$ is an intermediate $x_{1}$ coordinate direction.
The full transformation is then given by,

$$
\left[\begin{array}{l}
x_{1}^{\prime} \\
x_{2}^{\prime} \\
x_{3}^{\prime}
\end{array}\right]=\mathbf{T}\left[\begin{array}{l}
x_{1} \\
x_{2} \\
x_{3}
\end{array}\right]
$$

where $\mathbf{T}=\boldsymbol{\Psi} \boldsymbol{\Phi}$.

Consequently, the standard approach for transforming the fourth-order effective stiffness tensor $C_{i j k l}^{*}$, given the misalignment angles $\psi$ and $\phi$, is as follows,

$C_{i j k l}^{* /}=T_{i p} T_{j q} T_{k r} T_{l s} C_{p q r s}^{*}$

Referring to Eq. (6), it can be observed that, in the framework of micromechanics analysis, transforming the effective stiffness tensor $C_{i j k l}^{*}$ implies the transformation of,

$Z_{i j k l}^{(\beta \gamma)}=C_{i j p q}^{(\beta \gamma)} A_{p q k l}^{(\beta \gamma)}$

where $\mathbf{Z}^{(\beta \gamma)}=\left[Z_{i j k l}^{(\beta \gamma)}\right]$ can be referred to as a mixed concentration tensor, as it relates the subcell stresses to the global strains, see Eq. (7). Because this is a fourth-order tensor, its transformation is also given in the form of Eq. (13).

Eqs. (13) and (6) provide,

$C_{i j k l}^{* \prime}=\frac{1}{H L} \sum_{\beta=1}^{N_{\beta}} \sum_{\gamma=1}^{N_{\gamma}} h_{\beta} l_{\gamma} T_{i p} T_{j q} T_{k r} T_{l s} Z_{p q r s}^{(\beta \gamma)}=\frac{1}{H L} \sum_{\beta=1}^{N_{\beta}} \sum_{\gamma=1}^{N_{\gamma}} h_{\beta} l_{\gamma} Z_{i j k l}^{(\beta \gamma) /}$

In order to determine the local stresses in the subcells in the global coordinates including the effects of fiber misalignment, through the use of Eq. (7), one obtains,

$\bar{\sigma}_{i j}^{(\beta \gamma)}=Z_{i j k l}^{(\beta \gamma) /} \bar{\varepsilon}_{k l}$

The global stress-strain relations for the composite, including a fiber misalignment, can be expressed as,

$\bar{\varepsilon}_{i j}=S_{i j k l}^{* /} \bar{\sigma}_{k l}$

where $\mathbf{S}^{* /}=\left[\mathbf{C}^{* /}\right]^{-1}$. Substituting (17) into (16) leads to the final form of the local stresses in the subcells in the global coordinates, including the effects of fiber misalignment.

$\bar{\sigma}_{i j}^{(\beta \gamma)}=Z_{i j p q}^{(\beta \gamma) /} S_{p q k l}^{* /} \bar{\sigma}_{k l}=B_{i j k l}^{(\beta \gamma) /} \bar{\sigma}_{k l}$ 


\subsection{Probability-weighted averaging}

Now consider a known statistical distribution of fiber misalignment, for instance, over a composite part, coupon, or structure. Herein a normal fiber misalignment distribution has been adopted with probability density function given by,

$p(x)=\frac{1}{s \sqrt{2 \pi}} \exp \left[-\frac{1}{2}\left(\frac{x-\mu}{s}\right)^{2}\right]$

where $x$ is the random variable and $\mu$ and $s$ are the mean and standard deviation, respectively. Yurgartis [39] and Jelf and Fleck [16] have shown that local fiber misalignment angles in composites can be reasonably approximated by a normal distribution. Fig. 3 shows an example of a normal distribution probability density function vs. $x / s$ for the case where $\mu=0$.

In order to account for the known fiber misalignment variability with respect to angles $\psi$ and $\phi$, see Eqs. (10) and (11), the following operation has been employed,

$\left\langle Z_{i j k l}^{(\beta \gamma)}\right\rangle=\int_{-\pi}^{\pi} \int_{-\pi}^{\pi} Z_{i j k l}^{(\beta \gamma) /}(\psi, \phi) p(\psi) p(\phi) d \psi d \phi$

providing the probability-weighted average of the mixed concentration tensor. It should be noted that, in Eq. (20), the transformed mixed concentration tensor is a function of both transformation angles, $\psi$ and $\phi$. Therefore, Eq. (20) is valid for any choice of probability density functions for either angle.

Observing Eq. (15), it is clear that the probability-weighted average of the effective stiffness tensor of the composite is given by,

$\left\langle C_{i j k l}^{*}\right\rangle=\frac{1}{H L} \sum_{\beta=1}^{N_{\beta}} \sum_{\gamma=1}^{N_{\gamma}} h_{\beta} l_{\gamma}\left\langle Z_{i j k l}^{(\beta \gamma)}\right\rangle$

Finally, from Eq. (16), the subcell stresses in the presence of probability-weighted averaging, are given by,

$\bar{\sigma}_{i j}^{(\beta \gamma)}=\left\langle Z_{i j k l}^{(\beta \gamma)}\right\rangle \bar{\varepsilon}_{k l}$

The global stress-strain relations for the composite, including probability-weighted averaging, can be expressed using Eq. (17) as,

$\bar{\varepsilon}_{i j}=\left\langle S_{i j k l}^{*}\right\rangle \bar{\sigma}_{k l}$

where $\left\langle\mathbf{S}^{*}\right\rangle=\left\langle\mathbf{C}^{*}\right\rangle^{-1}$. Substituting Eq. (23) into Eq. (22) gives the final form of the subcell stresses, as follows,

$\bar{\sigma}_{i j}^{(\beta \gamma)}=\left\langle Z_{i j p q}^{(\beta \gamma)}\right\rangle\left\langle S_{p q k l}^{*}\right\rangle \bar{\sigma}_{k l}=\left\langle B_{i j k l}^{(\beta \gamma)}\right\rangle \bar{\sigma}_{k l}$

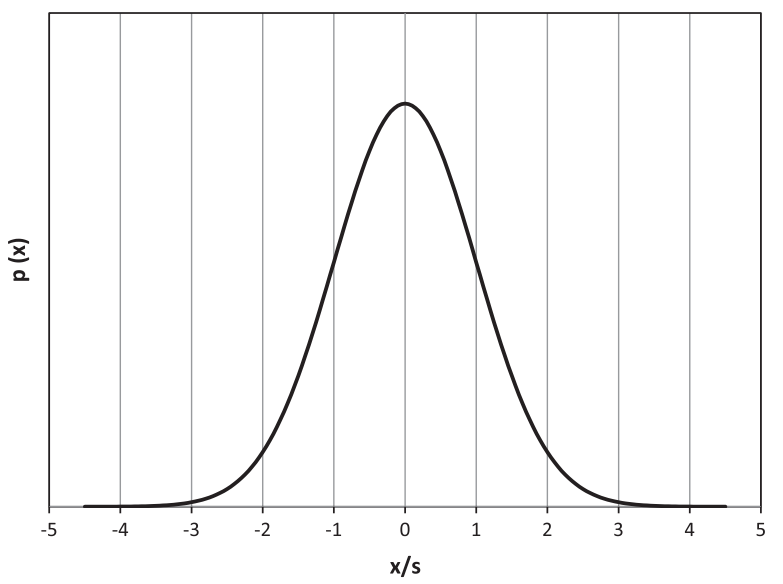

Fig. 3. Normal distribution probability density function, where the random variable has been normalized by the standard deviation and with the mean equal to zero.

\subsection{Fully random averaging}

In the case where the fibers within the composite are fully randomly oriented, the overall behavior of the composite is isotropic. The transformation of the mixed concentration tensor, $\mathbf{Z}^{(\beta \gamma)}$, can be carried out using the three Eulerian angles, $\psi_{E}, \phi_{E}$, and $\theta_{E}$, Aboudi et al. [2]. A fourth-order tensor transformation is applied to relate the original and transformed systems. In tensorial notation, this transformation is given by [5],

$Z_{i^{\prime} j^{\prime} k^{\prime} \ell^{\prime}}^{((\gamma \gamma)}=a_{i^{\prime} i} a_{j^{\prime} j} a_{k^{\prime} k} a_{\ell^{\prime}} Z_{i j k \ell}^{(\beta \gamma)}$

where $a_{i^{\prime} i}$ are given by

$$
\begin{aligned}
& a_{1^{\prime} 1}=\cos \psi_{E} \cos \theta_{E} \cos \phi_{E}-\sin \psi_{E} \sin \phi_{E} \\
& a_{1^{\prime} 2}=-\cos \psi_{E} \sin \phi_{E}-\sin \psi_{E} \cos \theta_{E} \cos \phi_{E} \\
& a_{1^{\prime} 3}=\sin \theta_{E} \cos \phi_{E} \\
& a_{2^{\prime} 1}=\cos \psi_{E} \cos \theta_{E} \sin \phi_{E}+\sin \psi_{E} \cos \phi_{E} \\
& a_{2^{\prime} 2}=-\sin \psi_{E} \cos \theta_{E} \sin \phi_{E}+\cos \psi_{E} \cos \phi_{E} \\
& a_{2^{\prime} 3}=\sin \theta_{E} \sin \phi_{E} \\
& a_{3^{\prime} 1}=-\cos \psi_{E} \sin \theta_{E} \\
& a_{3^{\prime} 2}=\sin \psi_{E} \sin \theta_{E} \\
& a_{3^{\prime} 3}=\cos \theta_{E}
\end{aligned}
$$

The mean value of $Z_{i^{\prime} j^{\prime} k^{\prime} \ell^{\prime}}^{(\beta)}$ for random orientation is given by,

$\left\langle Z_{i^{\prime} j^{\prime} k^{\prime} \ell^{\prime}}^{(\beta \gamma)}\right\rangle=\frac{1}{8 \pi^{2}} \int_{0}^{2 \pi} d \psi \int_{0}^{2 \pi} d \phi \int_{0}^{\pi} a_{i^{\prime}{ }^{\prime}} a_{j^{\prime} j} a_{k^{\prime} k} a_{\ell^{\prime} \ell} Z_{i j k \ell}^{(\beta \gamma)} \sin \theta \mathrm{d} \theta$

Therefore, the effective isotropic stiffness tensor of the composite with fully random fiber orientations is given by Eq. (21), and the subcell stresses are given by Eq. (24). Note that Christensen and Waals [12] used a fully random averaging approach, along with the concentric cylinder model, to predict the elastic properties of composites with random oriented fibers. Also, Luo and Daniel [23] used a fully random averaging approach in conjunction with the Mori and Tanaka [29] micromechanical method to predict the effective properties of nanocomposites.

\section{Practical application}

The equations presented above are useful for assessing the impact of fiber misalign on, not only the effective properties, but also the local fields within composite materials. Given the local stress and strain fields, initiation of damage or other detrimental nonlinear mechanisms can be predicted for use as estimates of the composite allowables. This section provides a brief step-bystep example of how the presented methodology can be applied, prior to the results, which are presented in the next section.

Given a composite material with known fiber volume fraction and constituent material properties, the first step in applying the presented methodology is to discretize the composite repeating unit cell into a number of subcells. For example, consider a unidirectional graphite/epoxy composite with a fiber volume fraction of 0.6 . The transversely isotropic graphite fiber properties are as follows: axial Young's modulus $=276 \mathrm{GPa}$, transverse Young's modulus $=15 \mathrm{GPa}$, axial shear modulus $=15 \mathrm{GPa}$, axial Poisson's ratio $=0.2$, and transverse Poisson's ratio $=0.2$. The isotropic epoxy matrix properties are: Young's modulus $=3.42 \mathrm{GPa}$ and Poisson's ratio $=0.34$. A 40 by 40 subcell discretization of the repeating unit cell can be considered, as shown in Fig. 4.

Next, the fiber misalignment present in the composite must be specified. Fully random fiber alignment is a special case, as described in Section 3.3. Otherwise, the methodology presented above considers a normal distribution of fiber misalignment, see Eq. (19). The mean, $\mu$, and standard deviation, $s$, of the in plane 


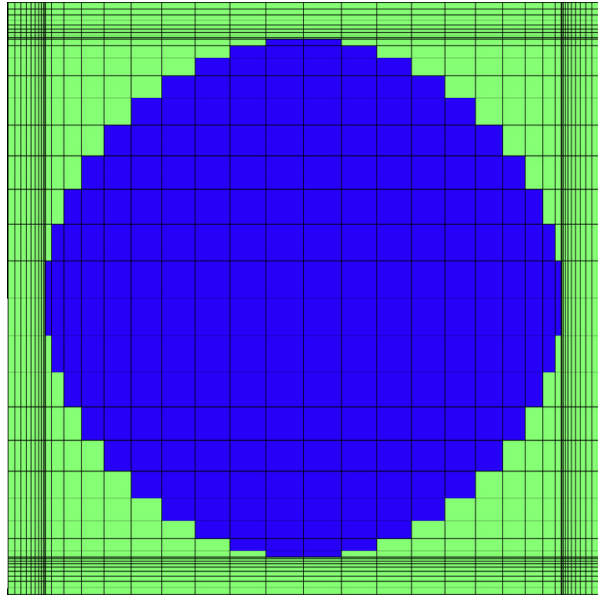

Fig. 4. Graphite/epoxy repeating unit cell divided into 40 by 40 subcells. The fiber volume fraction shown is 0.6 .

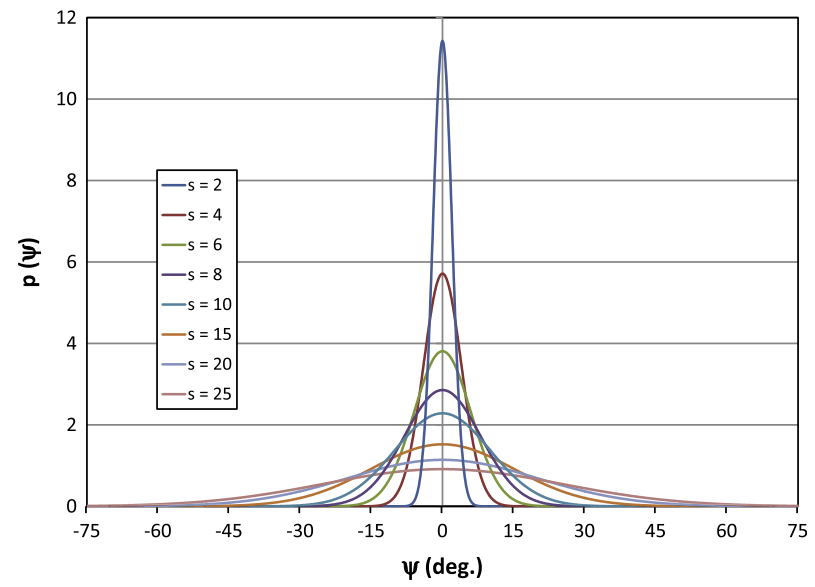

Fig. 5. Normal probability density functions vs. a misalignment angle are shown for various values of the standard deviation, $s$, where the mean is taken to be zero.

$(\psi)$ and out-of-plane $(\phi)$ fiber angles within the composite fully characterizes the fiber misalignment. For example, with the mean fiber angles representing perfectly aligned fibers, $\mu_{\psi}=\mu_{\phi}=0$, the standard deviations might be $s_{\psi}=3^{\circ}$ and $s_{\phi}=2^{\circ}$. Then, based on
Eq. (21), the effective elastic properties of the this misaligned composite can be predicted, as can the effective stresses throughout the composite, based on Eq. (24), given specified global composite level stresses. The results for the specified example are that the predicted effective axial Young's modulus of the composite is reduced from $164.7 \mathrm{GPa}$ (in the perfectly aligned case) to 163.2 GPa (reduction of $0.9 \%$ ), whereas, in response to a global uniaxial applied stress of $1 \mathrm{MPa}$ in the $0^{\circ}$ fiber direction, the maximum von Mises stress in the matrix increases from 21.3 Pa to 23.3 Pa (increase of 9.4\%). Hence, a slight reduction in axial modulus and a moderate reduction in the axial stress allowable might be warranted for use in design of such a composite with this fiber misalignment distribution.

\section{Results and discussion}

Results are shown for a polymer matrix composite (PMC) and a ceramic matrix composite (CMC) that exhibit the effects of fiber misalignment on the composite effective properties, local field distributions within the constituents, and the initial damage surfaces (envelopes). Both probability-weighted averaging of the misalignment and the fully random fiber orientation case are presented.

In order to generate the initial damage surfaces, two criteria were used, given the externally applied stresses, $\bar{\sigma}_{i j}$, or strains, $\bar{\varepsilon}_{i j}$, on the composite. The first is the traditional von Mises criteria, given by,

$\bar{\sigma}_{e q}^{(\beta \gamma)}=\sqrt{\frac{3}{2} \hat{\sigma}_{i j}^{(\beta \gamma)} \hat{\sigma}_{i j}^{(\beta \gamma)}}=Y$

where $\hat{\sigma}_{i j}^{(\beta \gamma)}=\bar{\sigma}_{i j}^{(\beta \gamma)}-\bar{\sigma}_{k k}^{(\beta \gamma)} \delta_{i j} / 3$ are the subcell deviatoric stresses, $\delta_{i j}$ is the Kronecker delta, and $Y$ is the yield stress in simple tension. The second damage initiation criterion, suggested by Lemaitre and Chaboche [20], is used to express the critical strain energy release rate associated with loss of stiffness in brittle materials, which are typically highly dependent on the state of triaxial stress, $\sigma_{h}=\sigma_{k k} /$ 3. The average triaxiality function [19] in subcell $(\beta \gamma)$ is given by,

$\bar{R}_{v}^{(\beta \gamma)}=\frac{2}{3}\left(1+v^{(\beta \gamma)}\right)+3\left(1-2 v^{(\beta \gamma)}\right)\left(\frac{\bar{\sigma}_{h}^{(\beta \gamma)}}{\bar{\sigma}_{e q}^{(\beta \gamma)}}\right)^{2}$

where $v^{(\beta \gamma)}$ is the Poisson's ratio of the isotropic subcell and $\bar{\sigma}_{h}^{(\beta \gamma)}$ is the subcell average hydrostatic pressure. The damage initiation criterion is then given by,

$\bar{\sigma}_{e q}^{(\beta \gamma)} \bar{R}_{v}^{(\beta \gamma) \frac{1}{2}}=\sigma_{c r}$
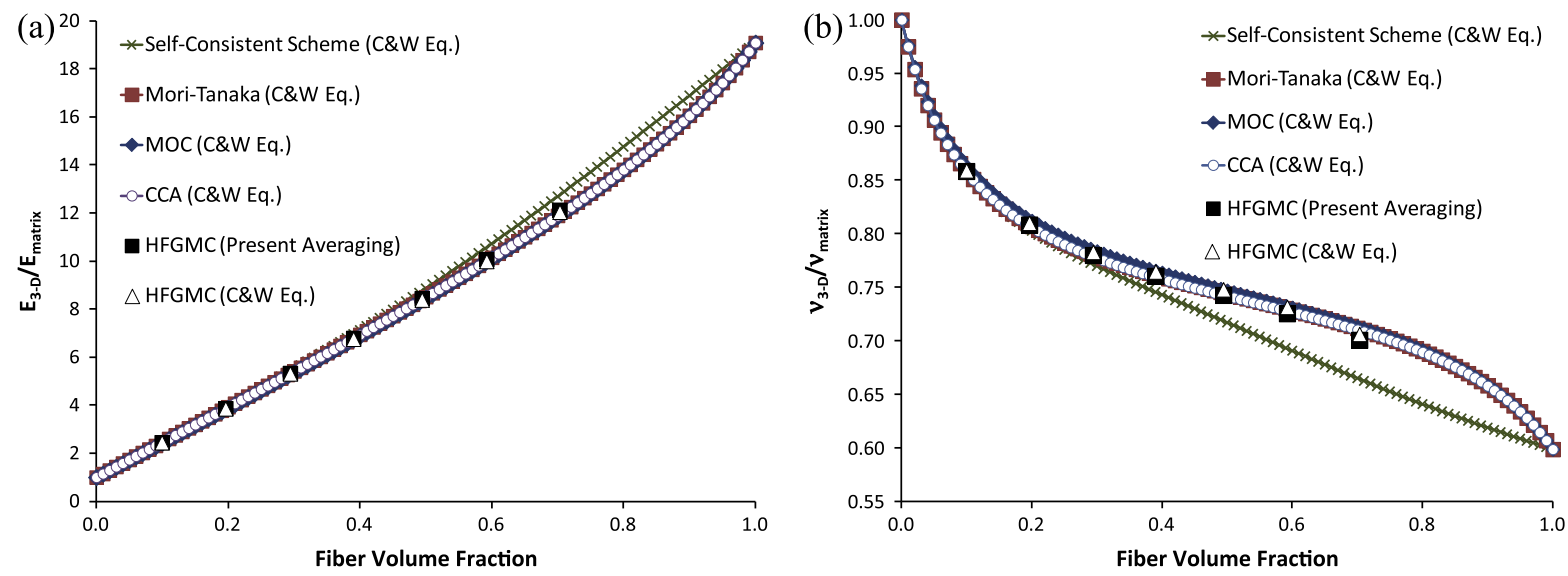

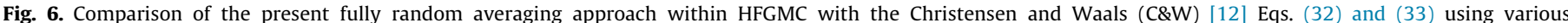

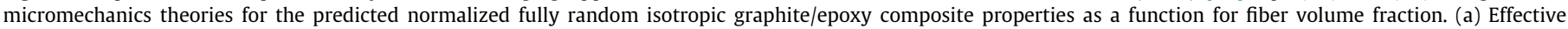
Young's modulus, E3-D. (b) Effective Poisson's ratio, $v_{3-D}$. 

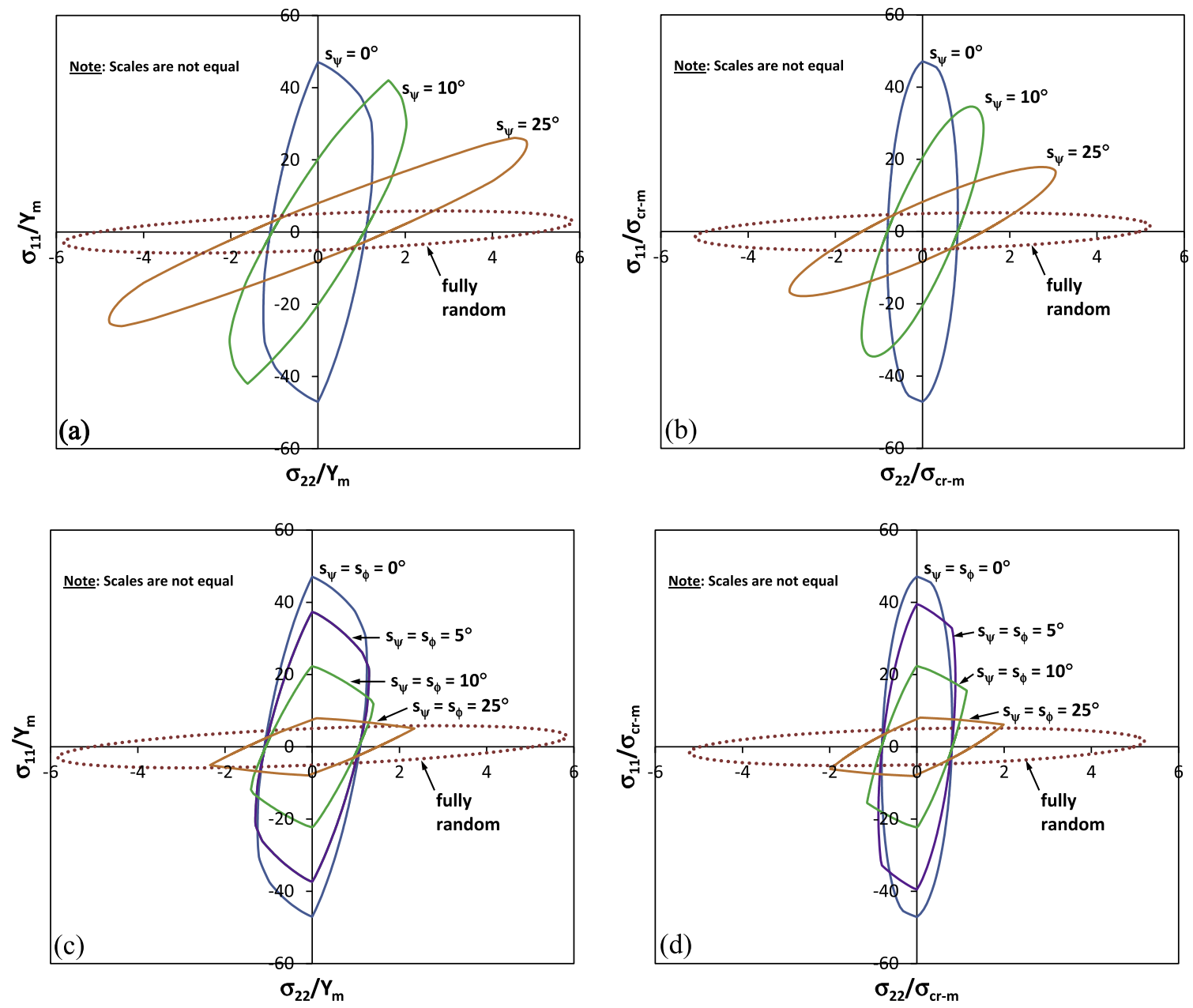

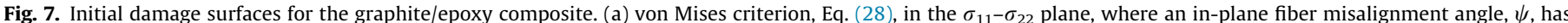

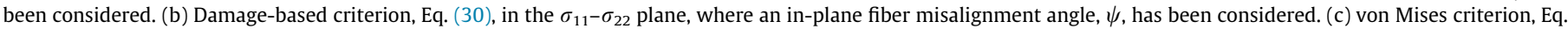

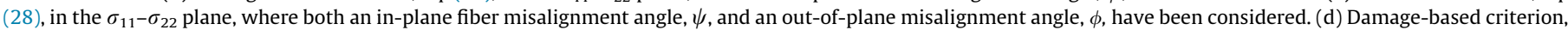
Eq. (30), in the $\sigma_{11}-\sigma_{22}$ plane, where both an in-plane fiber misalignment angle, $\psi$, and an out-of-plane misalignment angle, $\phi$, have been considered.
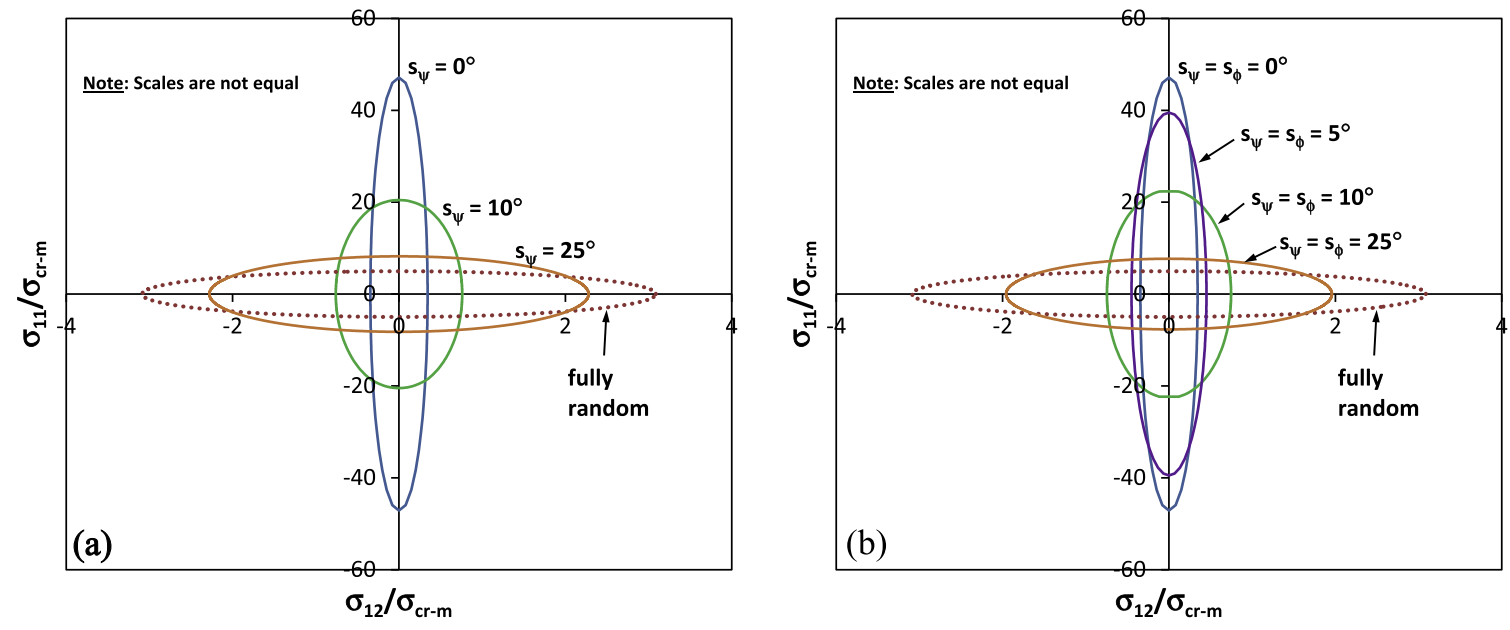

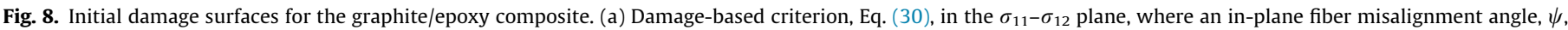

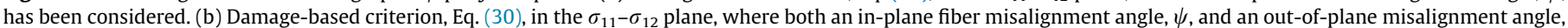
$\phi$, have been considered.

where $\sigma_{c r}$ is a material parameter that specifies the stress at which damage initiates for a uniaxial state test. Thus, if the triaxiality function is 1 and $\sigma_{c r}=Y$, this damage initiation criterion is equivalent to the von Mises criterion, Eq. (28). 
To generate a damage initiation surface in a given global stress plane, $\bar{\sigma}_{i j}-\bar{\sigma}_{k l}$ (e.g., $\bar{\sigma}_{11}-\bar{\sigma}_{22}$ ), the stress components are expressed as,

$\bar{\sigma}_{i j}=R \cos \alpha \quad \bar{\sigma}_{k l}=R \sin \alpha$

where $R$ is the radial distance from the origin at a point located on the initial damage surface in the $\bar{\sigma}_{i j}-\bar{\sigma}_{k l}$ stress plane and $\alpha$ is the corresponding polar angle. For a given $\alpha$, the local (subcell) stress components are readily determined (in terms of $R$ ) by substituting Eqs. (31) into Eq. (8). These expression for the local stresses can then be substituted into either initiation criterion, Eq. (28) or Eq. (30). This provides the value of $R / Y$ or $R / \sigma_{c r}$ corresponding to damage initiation at the specified polar angle $\alpha$.

As stated earlier, normal distributions for the fiber misalignment angles has been presently adopted, see Eq. (19). In order to perform the integration including the normal distribution in Eq. (20), finite limits of integration must be determined. Assuming that the average fiber misalignment angle (for both $\psi$ and $\phi$ ) is $\mu=0$, Fig. 5 shows the probability density functions for various value of the standard deviation, $s$. It is clear that it is sufficient to utilize $4 \mathrm{~s}$ as the limits of the infinite integrations in Eq. (20) since wider limits will have negligible contributions (see Fig. 3).

\subsection{Polymer matrix composite}

The first application considers the 0.6 unidirectional graphite/ epoxy composite described previously in Section 4. The same constituent properties and same 40 by 40 subcell discretization of the repeating unit cell, as shown in Fig. 4, have been considered.

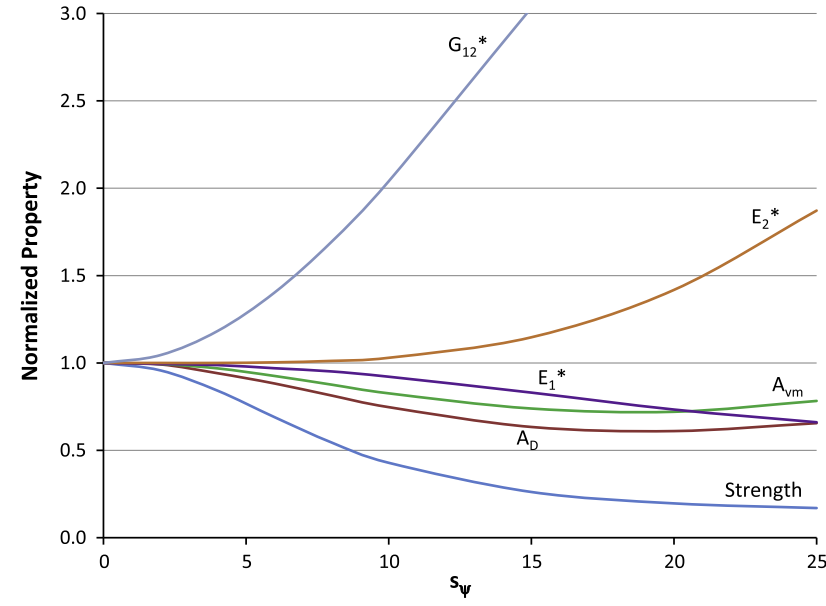

Fig. 9. Effect of in-plane fiber misalignment on the graphite/epoxy composite effective axial shear modulus $G_{12}^{*}$, transverse Young's modulus $E_{2}^{*}$, axial Young's modulus $E_{1}^{*}$, area of the von Mises criterion damage initiation envelope $A_{v m}$, area of the damage-based criterion damage initiation envelope $A_{D}$, and strength, all normalized with respect to their pristine values.

The HFGMC theory has been extensively validated vs. experiment data, and extensively verified vs. detailed finite element models, in both the linear and nonlinear regimes for polymer, metal, and ceramic matrix composites (c.f., [2,9,32,24]. In order to verify the fully random fiber orientation averaging procedure, described in Section 3.3, comparison has been made to results for
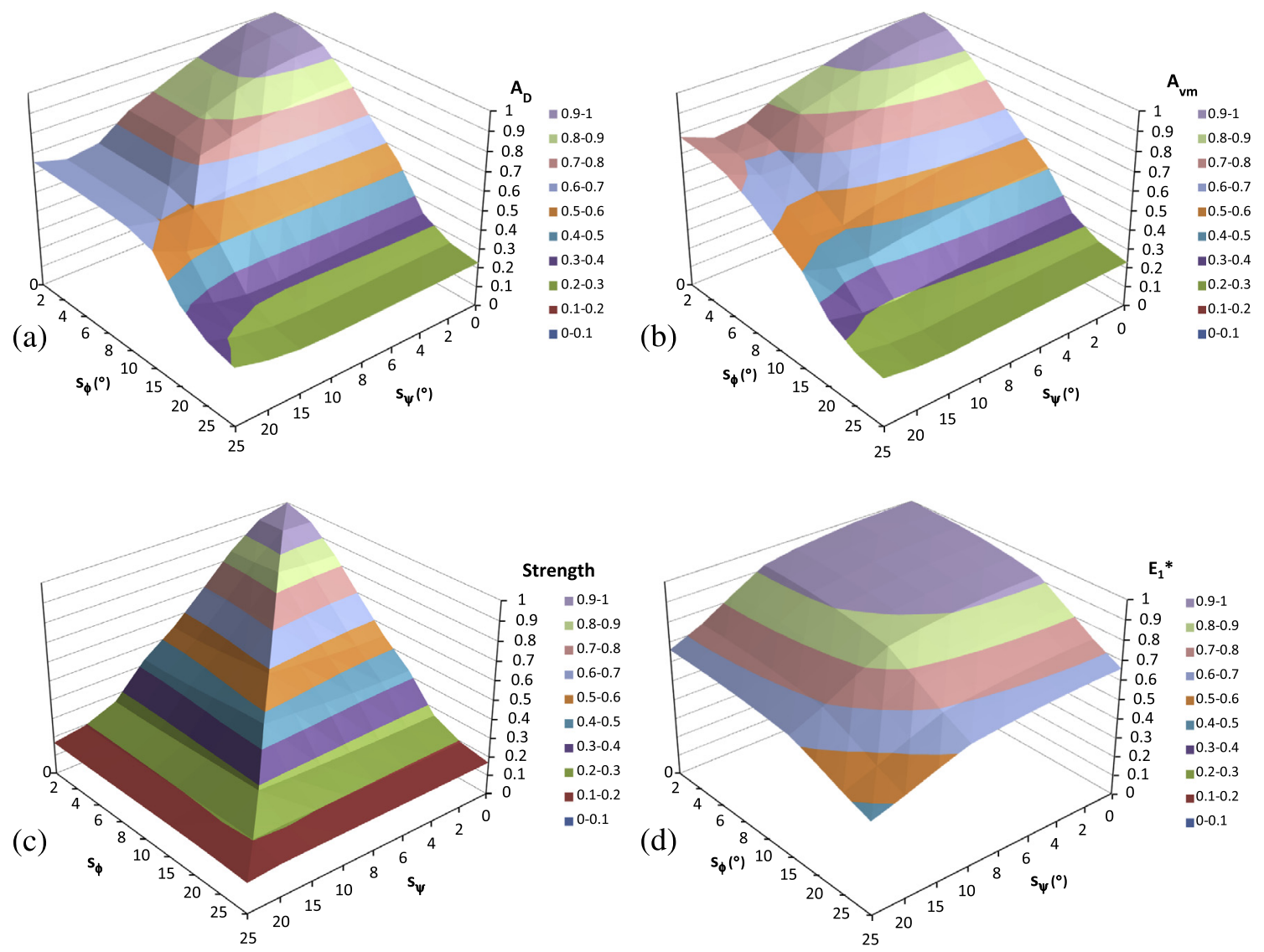

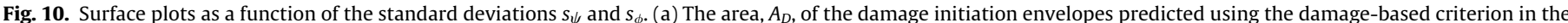

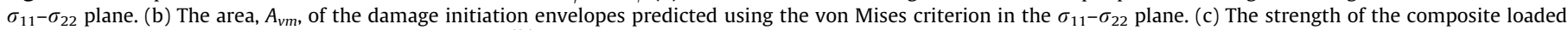
axially, as dictate by the maximum von Mises stress, $\bar{\sigma}_{e q}^{(\beta \gamma)}$. (d) The effective axial Young's modulus, $E_{1}^{*}$. 

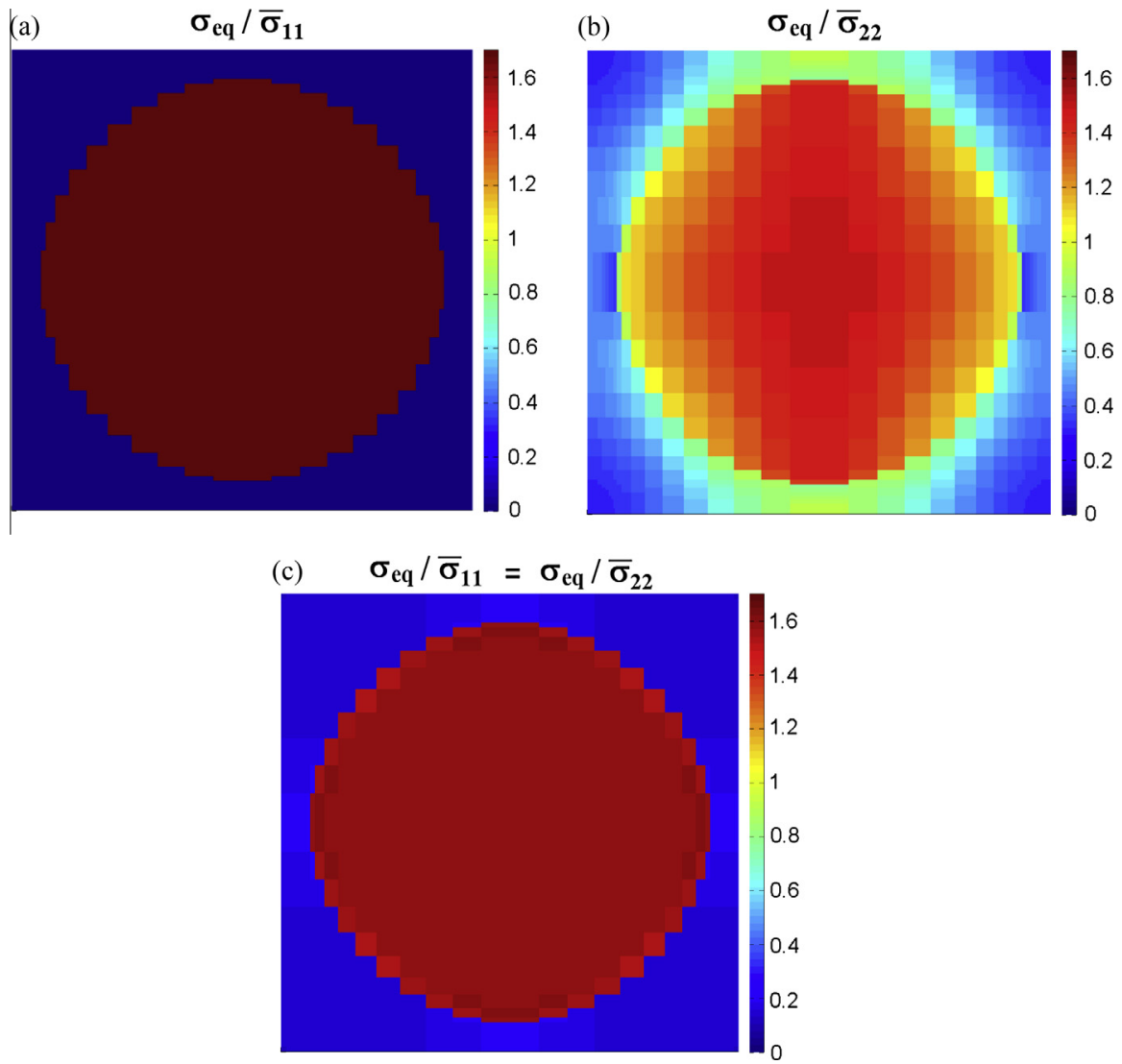

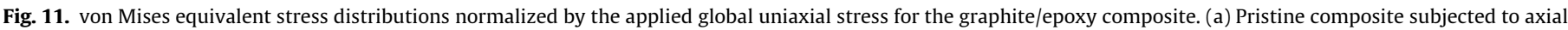
stress $\bar{\sigma}_{11}$. (b) Pristine composite subjected to transverse stress $\bar{\sigma}_{22}$. (c) Fully random fiber distribution subjected to either axial or transverse stress.

the effective Young's modulus and effective Poisson's ratio based on the equations presented by Christensen and Waals [12]. These equations are as follows,

$E_{3-D}=\frac{\left[E_{11}^{*}+\left(4 v_{12}^{* 2}+8 v_{12}^{*}+4\right) K_{23}^{*}\right]\left[E_{11}^{*}+\left(4 v_{12}^{* 2}-4 v_{12}^{*}+1\right) K_{23}^{*}+6\left(G_{12}^{*}+G_{23}^{*}\right)\right]}{3\left[2 E_{11}^{*}+\left(8 v_{12}^{* 2}+12 v_{12}^{*}+7\right) K_{23}^{*}+2\left(G_{12}^{*}+G_{23}^{*}\right)\right]}$

$v_{3-D}=\frac{E_{11}^{*}+\left(4 v_{12}^{* 2}+16 v_{12}^{*}+6\right) K_{23}^{*}-4\left(G_{12}^{*}+G_{23}^{*}\right)}{4 E_{11}^{*}+\left(16 v_{12}^{* 2}+24 v_{12}^{*}+14\right) K_{23}^{*}+4\left(G_{12}^{*}+G_{23}^{*}\right)}$

where $E_{11}^{*}, v_{12}^{*}, K_{23}^{*}, G_{12}^{*}$, and $G_{23}^{*}$ are the effective composite axial Young's modulus, axial Poisson's ratio, transverse plane strain bulk modulus, axial shear modulus, and transverse shear modulus, respectively. Note that these equations assume transverse isotropy, with five independent material constants. Christensen and Waals [12] used the concentric cylinder assemblage (CCA) model to predict the effective composite properties for use in Eqs. (32) and (33). Fig. 6 compares the predicted fully random (isotropic) effective graphite/epoxy composite properties predicted by the present fully random averaging approach implemented within HFGMC with the Christensen and Waals (C\&W) [12] Eqs. (32) and (33), as a function of fiber volume fraction, normalized by the isotropic matrix properties. In addition to the CCA model, predictions are shown for the Self-Consistent Scheme [15], the Mori and Tanaka [29] Method, the Method of Cells (MOC) [1], and HFGMC, wherein the C\&W averaging equations have been used. Note that, in the case of the CCA model, as done by Christensen and Waals [12], the lower bound estimates were used for the composite transverse properties. As shown, the present averaging approach matches closely with these other predictions. It should be noted, however, that these other, simpler micromechanics theories cannot provide a reliable estimate

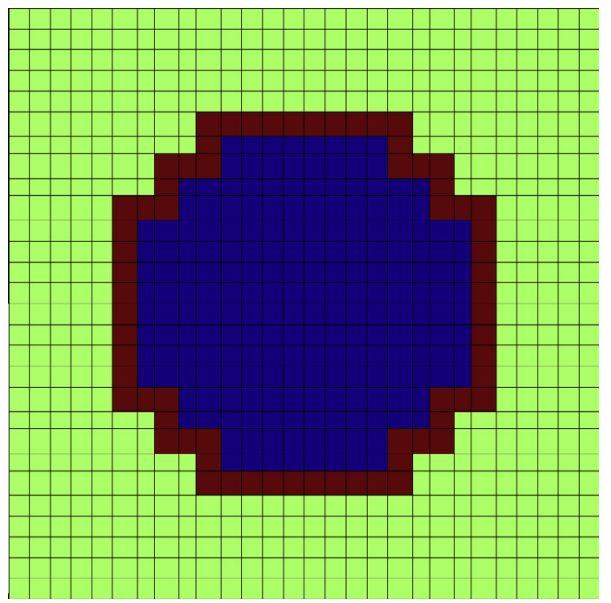

Fig. 12. $\mathrm{SiC} / \mathrm{SiC} \mathrm{CMC}$ repeating unit cell divided into 28 by 28 subcells. The fiber volume fraction is 0.26 and the interfacial layer volume fraction is 0.1 .

of the local field distributions, and they all lack shear coupling (see [2]. Slight ( $<1 \%)$ differences exist between the present fully random averaging approach and the $\mathrm{C} \& \mathrm{~W}$ averaging equations when using HFGMC with the square fiber packing arrangement represented by the employed repeating unit cell (see Fig. 4) because, as is well-known, such a packing arrangement leads to six rather than five independent material constants associated with transverse isotropy. As mentioned previously, the C\&W averaging equations assume transverse isotropy, whereas the present averaging approach includes no such assumption. While the present averaging 

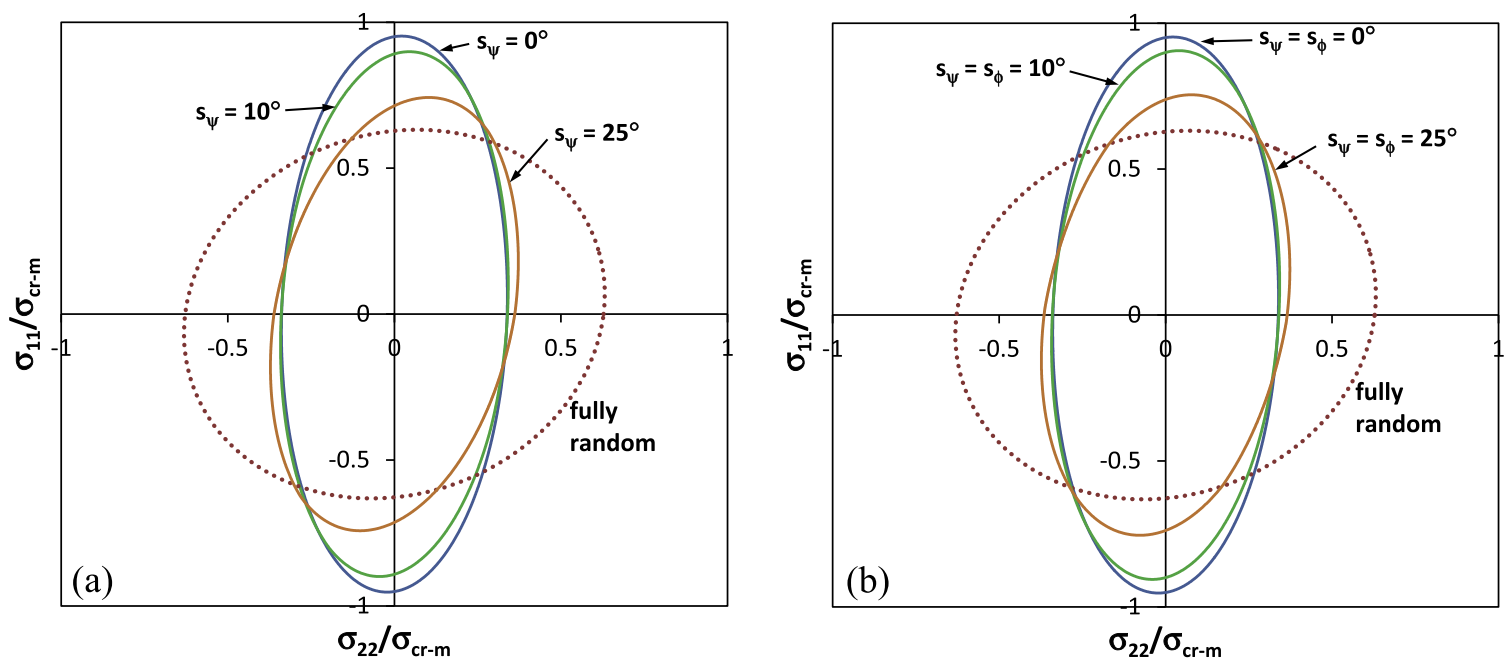

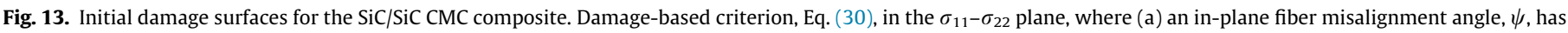
been considered, and (b) both an in-plane fiber misalignment angle, $\psi$, and an out-of-plane misalignment angle, $\phi$, have been considered.

approach, involving concentration tensors, produces accurate composite effective properties, the strength of the method involves its ability to predict local fields.

The remainder of the PMC results focus on the 0.6 volume fraction composite shown in Fig. 4. Fig. 7 exhibits the predicted initial damage surfaces for the von Mises criterion, Eq. (28), and the damage-based criterion, Eq. (30), for various values of the standard deviation of the misalignment angles, $s_{\psi}$ and $s_{\phi}$ under normal loading in the fiber $\left(x_{1}\right)$ and transverse $\left(x_{2}\right)$ directions. Also shown in the figure are the envelopes for the fully random case. In the absence of misalignment $(s=0)$, the envelopes exhibit the expected shape in that the strength in the fiber direction $\left(x_{1}\right)$ is more than 40 times that in the transverse $\left(x_{2}\right)$ direction. As can be seen in Fig. 7a and b, the effect of an in-plane misalignment angle, $\psi$, distribution, rotates the initial damage envelope such that the composite becomes weaker in the axial direction and stronger in the transverse direction. The fully random envelope, which represents an isotropic material, is nearly elliptical. The effect of the hydrostatic stress, which is included in the damage-based criterion through the triaxiality function, $R_{v}$, can be clearly observed by comparing Fig. 7a and b.

Fig. 7c and d shows the initial damage surfaces in the presence of equal in-plane, $\psi$, and out-of-plane, $\phi$, misalignment angle distributions. It can be seen that the effect of incorporating misalignment out of the $x_{1}-x_{2}$ plane is to reduce the size of the failure envelopes significantly as the reinforcing influence of the fiber is lost for both the axial and transverse directions.

Fig. 8 shows the initial damage surfaces for the damage based criterion in the $\sigma_{11}-\sigma_{12}$ plane for various values of the standard deviation of the misalignment angles. Also included is the fully random case. Note that the surfaces based on the von Mises criterion are not shown as they are quite similar to those shown in Fig. 8 since the hydrostatic stress effect is relatively small. In fact, the greatest value of the triaxiality function, $R_{v}$ (see Eq. (29)), throughout the matrix material for all points on all damage surfaces shown in Fig. 8 is 1.16 , whereas, for the damage surfaces shown in Fig. 7, this value is 5.15. It can be observed in Fig. 8 that the initial damage envelopes both rotate and reduce size as the fiber misalignment angle distribution standard deviations are increased. It should be noted that, contrary to the cases shown in Fig. 7, where additional fiber misalignment causes only minor strengthening in the transverse direction, in Fig. 8, it can be observed that significant longitudinal shear strengthening occurs with additional in-plane misalignment (as one might expect).
It is of interest to examine the variation of various composite properties with respect to the in-plane misalignment angle $\psi$. Fig. 9 shows a number of such properties, normalized with respect to their values in the absence of misalignment. This figure shows that, while the effective axial Young's modulus, $E_{1}^{*}$, decreases with additional misalignment, the transverse Young's modulus, $E_{2}^{*}$, and the axial shear modulus, $G_{12}^{*}$, increase. The axial and transverse Young's moduli are relatively insensitive to small amounts of misalignment, whereas the axial shear modulus is very sensitive. It is worth mentioning that, by applying an in-plane transformation, the initially orthotropic composite material becomes monoclinic and the effective stiffness tensor component $C_{1212}^{* /}$, see Eq. (13), is greatly influenced by this transformation. As a result of the employed averaging procedure, see Eq. (20), the additional monoclinic terms in the effective stiffness matrix vanish (assuming the mean misalignment angle, $\mu=0$ ), but the strong effect remains on $\left\langle C_{1212}^{*}\right\rangle$, which is the effective axial shear modulus, $G_{12}^{*}$. Note, however, that, as mentioned in the Introduction, the dependence of the effective elastic properties on the misalignment could also be determined using a macromechanical analysis (provided the presented probability-weighted averaging procedure were

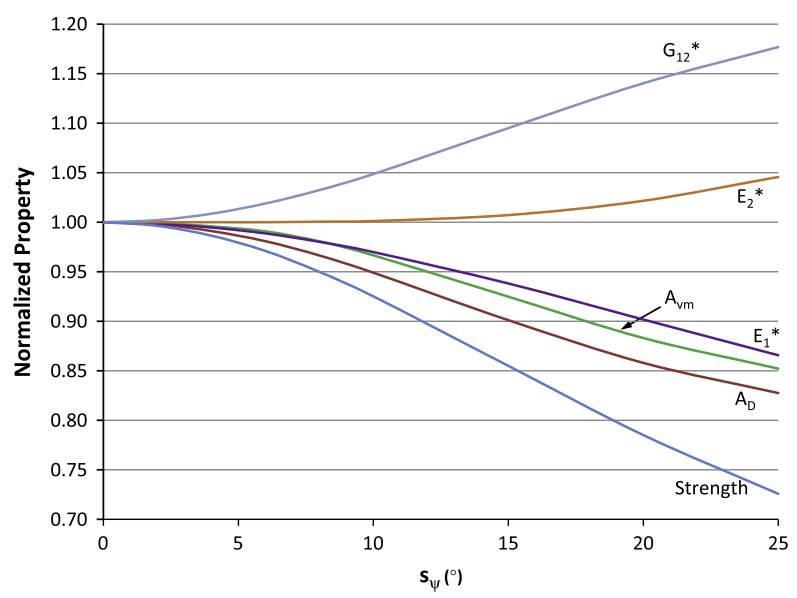

Fig. 14. Effect of in-plane fiber misalignment on the $\mathrm{SiC} / \mathrm{SiC} \mathrm{CMC}$ composite effective axial shear modulus $G_{12}^{*}$, transverse Young's modulus $E_{2}^{*}$, axial Young's modulus $E_{1}^{*}$, area of the von Mises criterion damage initiation envelope $A_{v m}$, area of the damage-based criterion damage initiation envelope $A_{D}$, and strength, all normalized with respect to their pristine values. 
performed). The influence on damage initiation surfaces, however, could not be determined.

Also shown in Fig. 9 are the variations of the areas of the damage initiation envelopes predicted using the von Mises, $A_{v m}$, and damage-based, $A_{D}$, criteria in the $\sigma_{11}-\sigma_{22}$ plane. The decreasing trends in these values indicate the overall loss of strength of the material in this plane due to fiber misalignment. However, for very large standard deviations of the misalignment angle, this trend appears to reverse.

A final curve plotted in Fig. 9 shows the impact of fiber misalignment on the predicted strength of the composite loaded in axial tension, as dictate by the maximum von Mises stress, $\bar{\sigma}_{e q}^{(\beta \gamma)}$, see Eq. (28), in the matrix constituent material. That is, this 'strength' corresponds to the global uniaxial tensile stress for damage initiation in the matrix, predicted based on the maximum local von Mises stress throughout the matrix. It can be readily observed that the impact of fiber misalignment on this axial strength, which can only be predicted by a micromechanical analysis, is substantial (for example, at $s_{\psi}=4^{\circ}, 16 \%$ reduction), and much greater than the impact on the axial Young's modulus, $E_{1}^{*}$ (for example, at $s_{\psi}=4^{\circ}$, only $1.2 \%$ reduction). Initially, as small amounts of misalignment are introduced, this curve decreases rapidly with increasing $s_{\psi}$ due to increased stress (particularly shear) in the matrix. At greater values of $s_{\psi}$, the normal distribution flattens (see Fig. 5), and the strength curve in Fig. 9 levels off, approaching the value for fully random fiber in the $x_{1}-x_{2}$ plane $(0.124)$.

In order to further investigate effect of the fiber misalignment on the composite, Fig. 10 shows surface plots of $A_{D}, A_{v m}$, the aforementioned von Mises stress based strength, and the effective axial Young's modulus $\left(E_{1}^{*}\right)$, all normalized by their pristine values as in Fig. 9. By adding the effect of out-of-plane misalignment, $\phi$, it can be observed that the effect of the latter misalignment is greater than that of in-plane misalignment on $A_{D}$ and $A_{v m}$ (Fig. 10a and b). As for the strength and effective axial Young's modulus, it appears that the effects of both in-plane and out-of-plane misalignments are similar. It is also clear that, across all values of both misalignment angle standard deviations, the effect on the effective axial Young's modulus, which could be calculated using the macroapproach, is significantly less than the other measures of composite performance. The fall off in the effective Young's modulus is also much less steep.

Fig. 11 shows the normalized von Mises equivalent stress distributions in the composite with no fiber misalignment (pristine) subjected to global uniaxial stress loading in the fiber and transverse directions. Also shown is the corresponding distribution for the case where the fibers are fully randomly oriented, whose response, as mentioned previously, is isotropic, which yields identical equivalent stress distributions irrespective of the orientation of the applied uniaxial loading. Comparing Fig. $11 \mathrm{a}-\mathrm{c}$, it is clear that the stresses in the fiber increase and the stresses in the matrix decrease by introducing random fiber orientations. The opposite result can be observed when comparing Fig. 11b and c. Thus the effect of random fiber orientation on the composite behavior is significant.

\subsection{Ceramic matrix composite}

A second application considers a 0.26 fiber volume fraction unidirectional $\mathrm{SiC} / \mathrm{SiC}$ composite. The isotropic SiC fiber properties are as follows: Young's modulus $=400 \mathrm{GPa}$ and Poisson's ratio $=0.17$. The isotropic SiC matrix properties are: Young's modulus $=335 \mathrm{GPa}$ and Poisson's ratio $=0.18$. In addition, there is an BN-based compliant interfacial layer whose volume fraction is 0.1. Its isotopic properties are as follows: Young's modulus $=5 \mathrm{GPa}$ and Poisson's ratio $=0.22$. A 28 by 28 subcell discretization of the (a)

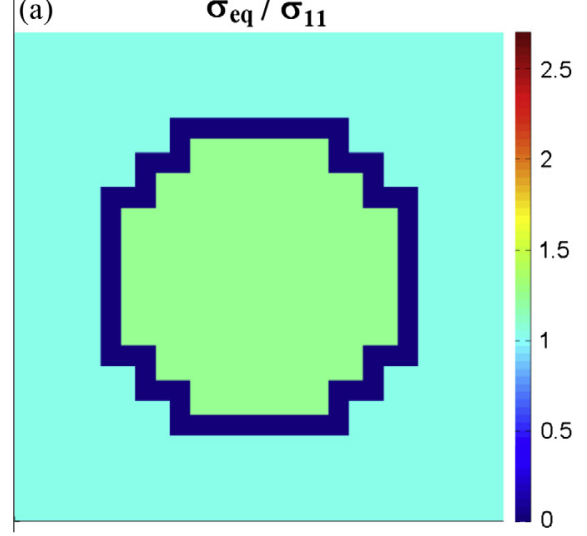

(b)

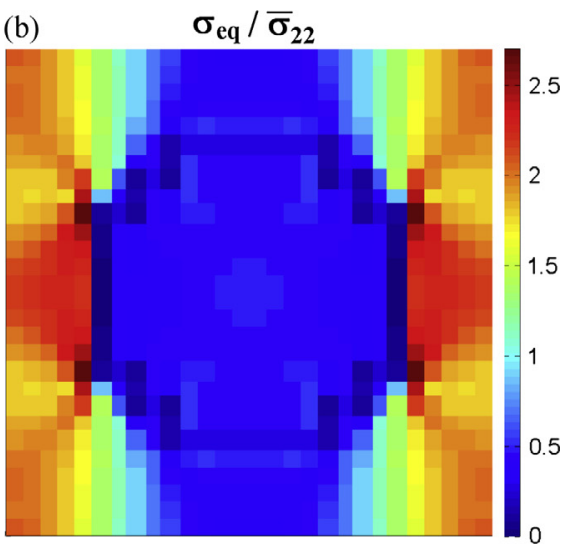

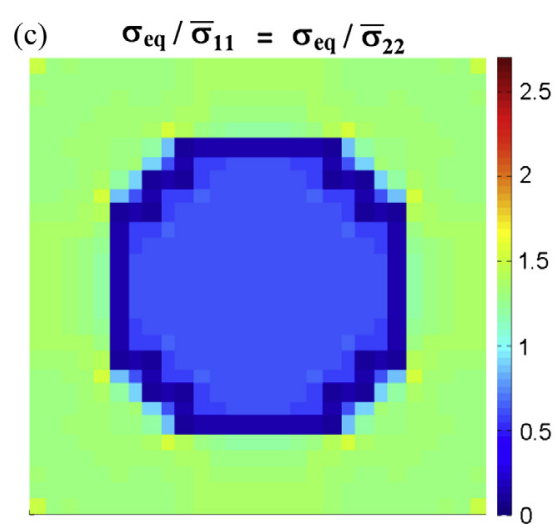

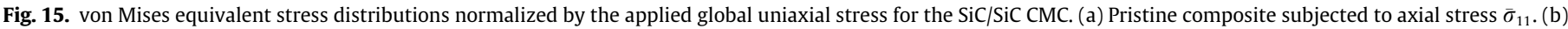
Pristine composite subjected to transverse stress $\bar{\sigma}_{22}$. (c) Fully random fiber distribution subjected to either axial or transverse stress. 
repeating unit cell, which now includes an explicit BN interfacial phase, has been considered, as shown in Fig. 12.

Fig. 13 shows the predicted initial damage surfaces for the CMC for the damage-based criterion, Eq. (30), for various values of the standard deviation of the misalignment angles, $s_{\psi}$ and $s_{\phi}$, under normal loading in the fiber $\left(x_{1}\right)$ and transverse $\left(x_{2}\right)$ directions. Also shown in the figure are the envelopes for the fully random case. The CMC behaves quite differently than the PMC previously considered. In the longitudinal (fiber) direction, the CMC exhibits much less mismatch in properties between the fiber and matrix, and the interfacial compliant layer, which is in parallel with the fiber and matrix, has only a minor effect. In contrast, in the transverse direction, the interfacial compliant layer (now in series with the fiber and matrix) introduces a great deal of property mismatch. This is exactly opposite to the PMC, wherein the longitudinal direction property mismatch it much larger (80:1) compared to the transverse mismatch (4:1). As a result, the effect of relatively small amounts of fiber misalignment on the CMC is considerably less compared to the PMC. Furthermore, also in contrast to the previously considered PMC, the addition of out-of-plane fiber misalignment, see Fig. 13b, does not exhibit a significant effect.

Fig. 14 shows the variation of various composite properties with respect to the in-plane misalignment angle $\psi$, normalized with respect to their values in the absence of misalignment. Note that the scale of the ordinate axis in Fig. 14 is significantly smaller than that in Fig. 9 for the PMC. As such, it is clear that the effect of misalignment on the various properties for the CMC is considerably less pronounced. The strength remains the property most affected by the fiber misalignment, but the trend reversals in the $A_{v m}$ and $A_{D}$, observed in Fig. 9 are now absent.

Finally, the normalized local von Mises equivalent stress distributions in the CMC are shown in Fig. 15. Fig. 15a and b shows the stress distributions in the pristine composite loaded in the axial and transverse directions, respectively. The compliant layer can be clearly observed. The induced stress concentrations are significantly higher in the transversely loaded case, Fig. 15b, due to the compliant layer's influence, which isolates the fiber. Fig. 15c shows the fully random $\mathrm{CMC}$ case, which, compared to the axially loaded pristine case, Fig. 15a, exhibits greater stress in the matrix and lower stress in the fiber.

\section{Conclusions}

A micromechanical formulation has been presented that is capable of predicting the effects of statistical distributions of fiber misalignment on the behavior of composite materials. The key unique feature is the ability of the method to predict not only effective composite properties, but also local fields. This is possible because this approach is based on the establishment of the composite concentration tensors, which include the effects of the fiber misalignment. It is thus possible (although not done herein) to predict the complete nonlinear response of the composite, including interfacial debonding and progressive damage. This approach was applied to the case of linear elasticity and perfect bonding, excluding progressive damage. However, in the absence of fiber misalignment, imperfect debonding and progressive damage have been addressed by Aboudi et al. [2]. In all cases presented, normal distributions of in-plane and out-of-plane fiber misalignment angles have been considered. It has been shown that the effect of these fiber misalignments are more significant in PMCs as compared to CMCs due to the fact that PMCs have significantly greater property mismatch. In the case of the PMC, the substantial variations of the initial damage surfaces with the standard deviation of the misalignment angles have been illustrated. Fully random fiber orientation has also been considered and shown to have a major impact on the initial damage surfaces and local field distributions when compared to the pristine composite.

It should be noted that the presented approach to capturing the effects of fiber misalignment is applicable to any micromechanical approach that can accurately predict the strain concentration tensor of a composite, including the finite element method. Due to the proven ability of the HFGMC model to analyze various nonlinear effects, the proposed method could also be extended to consider material nonlinearity such as damage and inelasticity within the constituents and to consider the thermal-mechanical response of composites. Additional potential applications include analysis of composites with given fiber paths, short/nanofiber composites with known fiber alignment/waviness (c.f., [38]) and multiscale analysis within laminated plate theories and finite element analyses, which could be used to capture misalignment effects on the response of woven composites. Application to biological materials, as has been done with the microsphere model, is also possible.

\section{References}

[1] Aboudi J. Micromechanical analysis of composites by the method of cells. Appl Mech Rev 1989;42:193-221.

[2] Aboudi J, Arnold SM, Bednarcyk BA. Micromechanics of composite materials a generalized multiscale approach. New York: Elsevier; 2013.

[3] Alastrué V, Martinez MA, Doblaré M, Menzel A. Anisotropic micro-spherebased finite elasticity applied to blood vessel modelling. J Mech Phys Solids 2009;57:178-203.

[4] Alastrué V, Sáeza P, Martinez MA, Doblaré M. On the use of the Bingham statistical distribution in microsphere-based constitutive models for arterial tissue. Mech Res Commun 2010:37:700-6.

[5] Arridge RGC. An introduction to polymer mechanics. London: Taylor and Francis; 1985.

[6] Ataabadia AK, Hosseini-Toudeshkyb H, Rada SZ. Experimental and analytical study on fiber-kinking failure mode of laminated composites. Compos B 2014;61:84-93.

[7] Barwick SC, Papathanasiou TD. Identification of fiber misalignment in continuous fiber composites. Polym Compos 2003:24:475-86.

[8] Basu S, Waas AM, Ambur DR. Compressive failure of fiber composites under multi-axial loading. J Mech Phys Solids 2006;54:611-34.

[9] Bednarcyk BA, Arnold SM, Aboudi J, Pindera M-J. Local field effects in titanium matrix composites subject to fiber-matrix debonding. Int J Plast 2004; $20: 1707-37$.

[10] Budiansky B. Micromechanics. Comput Struct 1983;16:3-12.

[11] Budianksky B, Fleck NA. Compressive failure of fibre composites. J Mech Phys Solids 1993;41:183-211.

[12] Christensen RM, Waals FM. Effective stiffness of randomly oriented fibre composites. J Compos Mater 1972;6:518-32.

[13] Göktepe S, Miehe C. A micro-macroapproach to rubber-like materials-Part III: the micro-sphere model of anisotropic Mullins-type damage. J Mech Phys Solids 2005;53:2259-83.

[14] Herakovich CT. Mechanics of fibrous composites. New York: John Wiley and Sons; 1998.

[15] Hill R. A self-consistent mechanics of composite materials. J Mech Phys Solids $1965 ; 13: 213-22$

[16] Jelf PM, Fleck NA. The failure of composite tubes due to combined compression and torsion. J Mater Sci 1994;29:3080-4.

[17] Jones RM. Mechanics of composite materials. New York: Hemisphere Publishing Corp; 1975

[18] Kugler D, Moon TJ. Identification of the most significant processing parameters on the development of fiber waviness in thin laminates. J Compos Mater 2001;36:1451-79.

[19] Lemaitre J. Handbook of materials behavior models. New York: Elsevier; 2001.

[20] Lemaitre J, Chaboche JL. Mechanics of solid materials. Cambridge: Cambridge University Press; 1990.

[21] Li Y, Simon J-W, Reese S. Microsphere-based homogenization for unidirectional fiber reinforced composites. In: 5th GACM colloquium on computational mechanics, Hamburg, Germany; 2013.

[22] Liu D, Fleck NA, Sutcliffe MPF. Compressive strength of fibre composites with random fibre waviness. J Mech Phys Solids 2004:52:1481-505.

[23] Luo JJ, Daniel IM. Characterization and modeling of mechanical behavior of polymer/clay nanocomposites. Compos Sci Technol 2003;63:1607-16.

[24] Matzenmiller A, Kurnatowski B. A comparison of micromechanical models for the homogenization of microheterogeneous elastic composites. In: Gilat R, Banks-Sills L, editors. Advances in mathematical modeling and experimental methods for materials and structures the Jacob Aboudi volume. New York: Springer; 2010.

[25] Menzel A, Waffenschmidt T. A microsphere-based remodelling formulation for anisotropic biological tissues. Philos Trans Roy Soc A 2009;367:3499-523. 
[26] Miehe C, Göktepe S, Lulei F. A micro-macroapproach to rubber-like materialsPart I: the non-affine micro-sphere model of rubber elasticity. J Mech Phys Solids 2004; 52:2617-60.

[27] Miehe C, Göktepe S. A micro-macro approach to rubber-like materials-Part II: the micro-sphere model of finite rubber viscoelasticity. J Mech Phys Solids 2005; $53: 2231-58$

[28] Modniks J, Andersons J. Modeling the non-linear deformation of a short-flaxfiber-reinforced polymer composite by orientation averaging. Compos B 2013:54:188-93.

[29] Mori T, Tanaka K. Average stress in matrix and average energy of materials with misfitting inclusions. Acta Metall 1973;21:571-4.

[30] Murtada SI, Kroon M, Holzapfel GA. Modeling the dispersion effects of contractile fibers in smooth muscles. J Mech Phys Solids 2010;58:2065-82.

[31] Numayr KS, Al Rjoub YS. Two analogous methods for estimating the compressive strength of fibrous composites. Compos B 2013;30:290-6.

[32] Pineda EJ, Bednarcyk BA, Waas AM, Arnold SM. Progressive failure of a unidirectional fiber-reinforced composite using the method of cells: discretization objective computational results. Int J Solids Struct 2013;20:1203-16.
[33] Schultheisz CR, Waas AM. Compressive failure of composites, Part I: testing and micromechanical theories. Prog Aerosp Sci 1996;32:1-42.

[34] Shuart MJ. Failure of compression-loaded multidirectional composite laminates. AIAA J 1989;27:1274-89.

[35] Waas AM, Schultheisz CR. Compressive failure of composites, Part II: experimental studies. Prog Aerosp Sci 1996;32:43-78.

[36] Waffenschmidt T, Menzel A, Kuhl E. Anisotropic density growth of bone-a computational micro-sphere approach. Int J Solids Struct 2012;49:1928-46.

[37] Wisnom MR. The effects of fibre misalignment on the compressive strength of unidirectional carbon fibre/epoxy. Composites 1990;21:403-7.

[38] Yu J, Lacy TE, Toghiani H, Pittman Jr CU, Hwang Y. Classical micromechanics modeling of nanocomposites with carbon nanofibers and interphase. J Compos Mater 2011:45:2401-13.

[39] Yurgartis SW. Measurement of small angle fiber misalignments in continuous fiber composites. Compos Sci Technol 1987;30:279-93.

[40] Zhou HW, Yi HY, Gui LL, Dai GM, Peng RD, Wang HW, et al. Compressive damage mechanism of GFRP composites under off-axis loading: experimental and numerical investigations. Compos B 2013;55:119-27. 